\title{
COVID-19 EN ESTADOS UNIDOS: LA DESIGUALDAD SOCIAL COMO CAUSA Y CONSECUENCIA
}

\section{COVID-19 in the United States: Social Inequality as Cause and Consequence}

\begin{abstract}
Usama BILAL Health, Drexel University, Philadelphia (USA).

Correo electrónico: ub45@drexel.edu

Fecha de recepción: 21 de septiembre de 2020

Fecha de aceptación: 27 de septiembre de 2020

Fecha de publicación: 29 de enero de 2021
\end{abstract}

Urban Health Collaborative \& Department of Epidemiology and Biostatistics, Drexel Dornsife School of Public

\section{Resumen}

La pandemia de COVID-19 es una de las crisis sanitarias globales mas importantes del último siglo. En este articulo resumimos la situación en los Estados Unidos de América (EEUU) con respecto a la pandemia, con una mezcla de observaciones personales y datos epidemiológicos. Reseñamos la importancia de considerar la situación sanitaria y política previa a la pandemia, desde donde EEUU parte como un país con una esperanza de vida más baja que otros países occidentales, con un sistema sanitario fragmentado y muy desigual. Seguidamente describimos como ha evolucionado la pandemia en el país, con un inicio en Nueva York y otras ciudades de la costa este, seguida de una segunda ola en el sur del país. Por último, destacamos dos retos fundamentales durante la pandemia: el agravamiento de las desigualdades en salud, y la situación política del país, que ha partidizado las medidas de control epidemiológico.

Palabras clave: COVID-19; desigualdades; equidad en salud; EEUU.

\section{Abstract}

The COVID-19 pandemic is one of the most severe health crises of the last century. In this manuscript, we summarize the epidemiologic situation of the United States of America (US), with 
a mixture of personal observations and epidemiological data. We highlight the importance of considering the pre-pandemic health and political situation, where the US showed a worse health status as compared to other Western economies, with an unequal and fragmented health system. We follow with a description of the evolution of the pandemic in the US, starting in New York and other large East Coast cities, followed by a second wave in the South. Last, we highlight two challenges during the pandemic: the worsening of existing health disparities and the political situation of the country, that has polarized epidemic control measures.

Keywords: COVID-19; inequalities; health equity; USA.

\section{INTRODUCCIÓN}

La enfermedad por coronavirus 2019 (COVID-19), causada por el virus SARS-CoV-2, ha puesto el mundo patas arriba. Desde los primeros casos hasta el presente (22 de septiembre de 2020), han sido diagnosticadas más de 31 millones de personas y han muerto casi 1 millón de estas en todo el mundo ${ }^{1}$, un numero muy subestimado por la limitada capacidad diagnostica en muchos países. En España, se han registrado casi 700,000 casos y más de 30,000 muertos ${ }^{1}$, aunque los análisis utilizando el exceso de mortalidad total ya muestran que esta cifra podría subir hasta más de los 40,000 muertos. Hasta el momento, en EEUU se han registrado casi 7 millones de casos y más de 200,000 fallecidos confirmados $^{1}$. Como en España, esta cifra probablemente sea mucho más alta. En este articulo quiero resumir la evolución y consecuencias de esta pandemia en EEUU. Específicamente, mezclando datos epidemiológicos con una serie de impresiones personales, quiero centrarme en: (1) el contexto previo a la pandemia; (2) la evolución epidemiologia de la pandemia en tiempo y espacio; (3) la aparición y agravamiento de las desigualdades sociales; y (4) los retos a los que se enfrenta el país en la respuesta a la pandemia.

\section{CONTEXTO PRE-PANDÉMICO}

EEUU es un país aproximadamente 7 veces más grande en cuanto a población y 20 veces más grande en cuanto a superficie. Es un país enormemente heterogéneo física y socialmente. A pesar de ser la primera economía mundial y uno de los países con mayor producto interior bruto per cápita del mundo, se trata de un país enormemente desigual, con un coeficiente Gini muy superior al de España y otros países europeos $^{2}$. Uno de los lugares donde se nota esta desigualdad es en la salud. Según datos del programa de desarrollo de las naciones unidas (PNUD), mientras que la esperanza de vida en España para ambos sexos para el periodo 20152020 fue de 83.4 años, esta fue de 78.8 años en EEUU, inferior a la de países como Chile y Eslovenia, y similar a Cuba y Líbano ${ }^{3}$. De hecho, como podemos ver en la Figura 1, a pesar de tener una esperanza de ida superior a muchos países europeos a mediados de siglo, EEUU se ha quedado atrás desde los años 80-90. Las causas de esta peor salud en EEUU son múltiples, incluyendo un sistema de protección social muy deficiente, enormes desigualdades sociales, y un sistema sanitario ineficiente e injusto ${ }^{4}$. Son precisamente estos mismos factores lo que nos lleva a la situación actual con el COVID-19.

El otro aspecto importante para entender la situación en EEUU es el contexto político. Tras la victoria de Donald Trump en las elecciones de 2016, se ha producido un giro marcado hacia una narrativa antiinmigración y contra minorías (étnicas, sexuales, etc.), y una disminución en aspectos regulatorios, especialmente en la Agencia de Protección Ambiental (EPA por sus siglas en ingles). En medio de estos cambios, la situación 


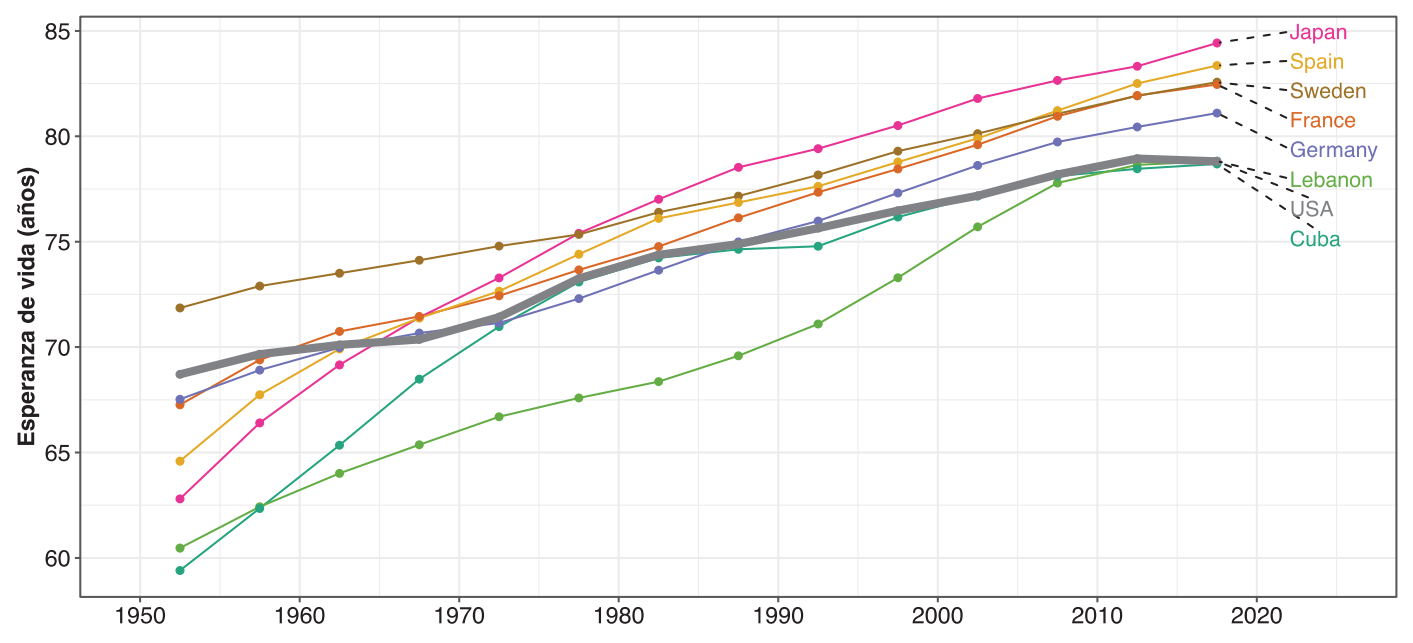

Figura 1. Esperanza de vida al nacer en ambos sexos en EEUU y otros 7 paises

Fuente: Programa de las Naciones Unidas para el Desarrollo ${ }^{3}$

en salud ha continuado empeorando, con una epidemia de obesidad que no cede, y una epidemia de consumo de opiáceos que mato a unas 70.000 personas solo en 2018. De hecho, esta epidemia de consumo de opiáceos coexiste con aumentos en otras causas de muerte, como la cirrosis alcohólica o los suicidios, que agrupadas todas ellas suelen denominarse las "muertes de la desesperación" (deaths of despair en inglés). Como hemos mostrado en un artículo publicado en 2019, estas muertes aumentaron mas aun en las zonas con mayor inseguridad económica y precariedad $^{5}$. Visto esto, la situación en salud y la victoria de Trump no están desconectadas. En otro artículo que publicamos en 2018 mostramos como las zonas donde más había aumentado la mortalidad en los años previos a las elecciones, y las zonas con mayores desigualdades en salud, habían sido las zonas que más habían aumentado la proporción de voto hacia Trump, con respecto a elecciones anteriores ${ }^{6}$. En esta situación de gobierno en retirada, mensajes de odio y empeoramiento de la salud de la población, llega el COVID-19.

\section{LA DESIGUALDAD SOCIAL PRE-PANDÉMICA}

Como mostrábamos al principio, EEUU es uno de los países mas desiguales dentro de las economías occidentales. Esta desigualdad no se vertebra solo en un aspecto puramente económico, sino que es también la consecuencia del racismo estructural sobre el que se creó el país. Durante 250 anos (1619 hasta 1865), la esclavitud fue uno de los principales motores económicos del país ${ }^{7}$, especialmente en los estados del sur. Esta esclavitud se ligó al concepto de raza, que se utilizó como justificación moral para poder llevar a cabo esta esclavitud. Con el fin de la esclavitud llegaron otras leyes, generalmente agrupadas bajo las leyes Jim Crow, que mantenían la segregación entre blancos y negros. Mientras que algunas de estas leyes generaban de forma muy explicita tal segregación (separación en transporte publico o restaurantes), algunas otras dejaron una herencia económica oculta, pero 
de enorme importancia. No fue hasta el movimiento de derechos civiles, que culmino con la ley de derechos civiles de 1964, que muchas de estas leyes se eliminaron, con un enorme poso que todavía vemos en estos momentos.

Mucha de la segregación racial que se observa hoy en día en ciudades estadounidenses puede trazarse hasta el concepto de redlining ${ }^{8}$. Una de las políticas del New Deal para salir de la gran depresión fue favorecer la compra de casas mediante hipotecas publicas a bajo coste. Recibir una de estas hipotecas dependía, principalmente, de la zona donde se compara la casa. Con esto, se crearon unos mapas de "deseabilidad" de cada zona. Si la zona tenía buenas condiciones económicas, se concedía la hipoteca, de no tenerlas, se denegaba. Estas zonas se coloreaban en un mapa, siendo el rojo las zonas menos deseables, de ahí el termino redlining. Uno de los principales factores que marcaban que una zona fuese roja era la presencia de minorías en tal zona, especialmente negros. La población negra, por tanto, no podía comprar casas en las zonas donde vivían por no ser "deseables" y, por otras de leyes y acuerdos privados, no se les permitían comprar casa en zonas "deseables". Esto tuvo dos consecuencias que todavía vivimos en el presente. Por un lado, la creación de una segregación residencial enorme, con ciudades donde la población negra y blanca viven en zonas muy separadas. Por otro lado, creó un enorme gradiente de riqueza, dado que la vivienda es uno de los bienes de mayor valor para familias de clase trabajadora. Al permitir que la población blanca comprara viviendas con hipotecas baratas, pero no lo hiciera la población negra, se creo un clivaje de riqueza entre los dos grupos que todavía sigue existente. Hoy en día, una persona blanca media tiene una riqueza estimada de $\$ 171.000$ dólares, mientras que este numero desciende a $\$ 17.000$ dólares en el caso de una persona negra 9 .

\section{EVOLUCIÓN DE LA PANDEMIA EN EEUU}

El coronavirus llego a EEUU por la costa oeste, probablemente vinculada a la gran conexión de vuelos entre esa zona del país y Asia. California y el estado de Washington, especialmente en Seattle, vivieron algunos de los primeros brotes. Pero no fue allí donde la pandemia tuvo sus más graves consecuencias, sino en la ciudad de Nueva York. El influjo de turistas y viajeros de negocios, sus conexiones con Europa, los problemas de vivienda y hacinamiento, todo ello hizo de Nueva York un lugar ideal para la extensión del virus. La Figura 2 muestra la evolución del numero de casos confirmados por laboratorio en las 10 áreas metropolitanas más grandes de EEUU. Nueva York destaca por su temprano inicio y su rápido aumento de casos.

Este aumento de casos en Nueva York se reflejó en una enorme crisis de mortalidad. En una investigación publicada en agosto de 2020, unos investigadores compararon el numero de muertes en el pico de mortalidad de la gripe de 1918 vs el numero de muertes en la crisis actual, encontrando que las diferencias eran pequeñas ${ }^{10}$. Es decir, para Nueva York el COVID-19 no fue tan diferente en cuanto a su impacto que la devastadora gripe de 1918. En la Figura 3 comparamos los datos de la ciudad de Nueva York con los de la Comunidad de Madrid, mostrando un aumento similar en ambas zonas, siendo incluso superior en Nueva York. En una investigación que publicamos recientemente, observamos que el exceso de mortalidad en Madrid llevo a caídas de esperanzas de vida semanal de hasta 12 años, con lo que esperamos que estas mismas caídas en Nueva York sean aun más impactantes ${ }^{11}$.

La respuesta ante la pandemia ha seguido un patrón similar al de otros países. Declaraciones de estado de emergencia, cierre de centros educativos y de trabajo, limitación de negocios a aquellos esenciales, etc. Quizás la diferencia más grande con respecto a la respuesta en España es la de la falta de un confinamiento como tal. Mientras

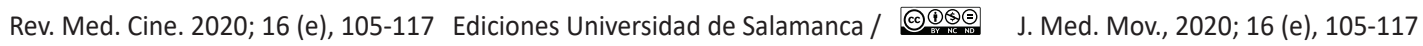
[ 108 ] 


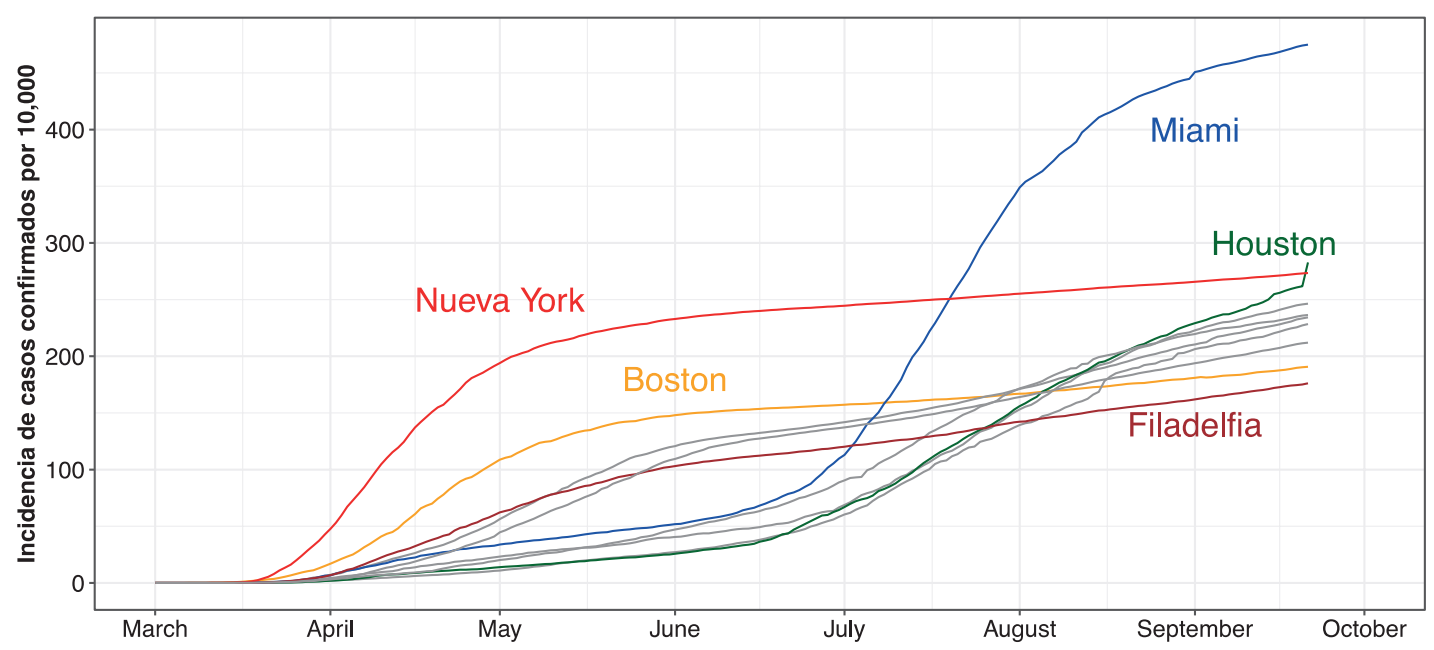

Figura 2. Incidencia acumulade de COVID-19 en 10 areas metropolitanas de EEUU Fuente: Johns Hopkins University ${ }^{1}$

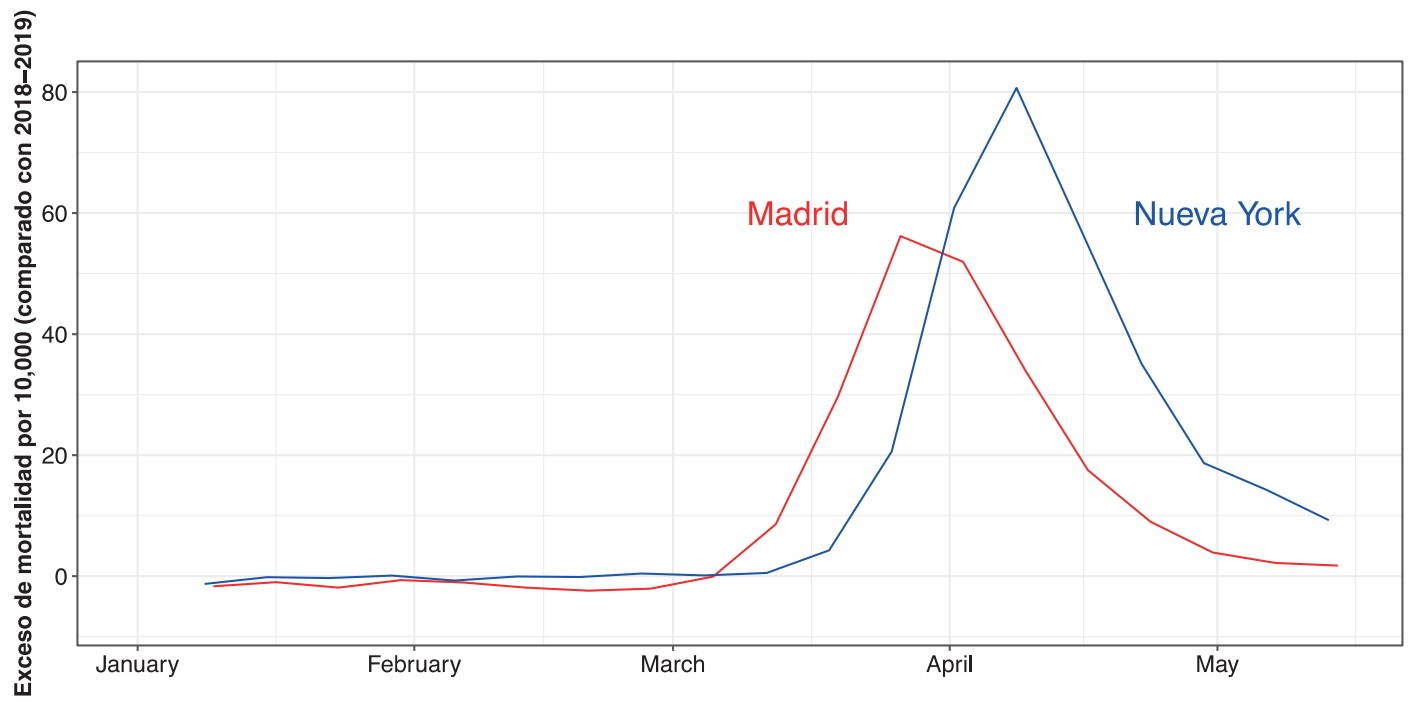

Figura 3. Exceso de mortalidad en Madrid (CCAA) y Nueva York (ciudad) Fuente: $\mathrm{MoMo}^{12}$ y National Center for Health Statistics ${ }^{13}$

que muchos estados y ciudades declararon lo que aquí se denomina como shelter-at-home orders, es decir, la necesidad de quedarse en casa, estas eran muy limitadas en comparación con el confinamiento en el que estuvo España. Un aspecto muy diferencial con España es el uso de espacios abiertos, que prácticamente nunca se limitó. Hacer deporte u otras actividades al 
aire libre supone, como ya sabemos ahora, una actividad de bajo riesgo ${ }^{14}$. Otra gran diferencia fue la velocidad de reapertura, especialmente la heterogeneidad en esta reapertura. En concreto, al momento de escribir este artículo (septiembre 2020) en Filadelfia acabamos de permitir el uso del interior de restaurantes, mientras que en Nueva York todavía sigue limitado. Al contrario, en ciertos estados del sur incluyendo Tejas, Florida y Luisiana, esta reapertura ocurrió ya de forma total en mayo. Pocas semanas más tarde, en junio, las consecuencias de esta reapertura temprana quedaron claras.

Como podemos ver en la Figura 4, a principios de junio empieza una gran subida de casos en todo el sur del país, centrada especialmente en esos 3 estados que fueron parte de esa primera reapertura en mayo. Fue como parte de esta segunda oleada cuando observamos uno de los mayores ejemplos de la importancia política de los datos en salud. Específicamente, según Florida empezaba a reabrir su economía, la persona encargada de actualizar el panel de datos publico del departamento de salud de Florida fue despedida por negarse a manipular los datos públicos para favorecer el plan de reapertura ${ }^{16}$.

Esta visión general de la pandemia en el tiempo y el espacio oculta varios aspectos que nos permitan entenderla de forma completa. En las siguientes dos secciones nos centraremos primero en las desigualdades sociales durante el COVID-19, y luego en la situación política durante el tiempo de pandemia.

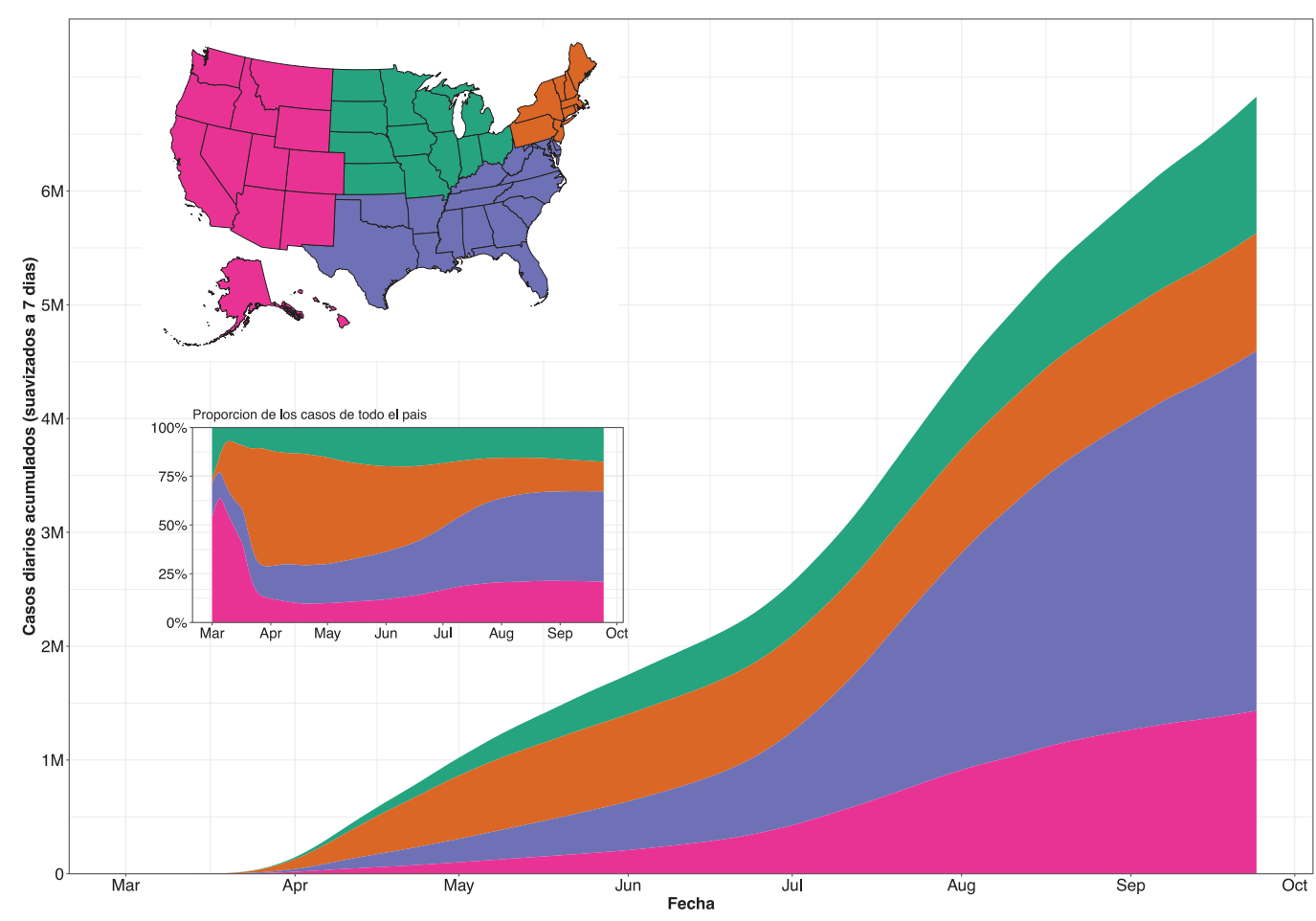

Figura 4. Casos por Region en EEUU

Fuente: New York Times COVID-19 Data ${ }^{15}$ 


\section{DESIGUALDADES SOCIALES DURANTE EL COVID-19}

Muy al principio de la pandemia, una vez estaba claro que su impacto iba a ser alto en la población en general, empezó a circular un rumor por las redes sociales: la población negra era inmune al COVID-19. Claramente falso, lograr desarticular este rumor requirió por un lado de personalidades como Idris Elba (Stringer Bell en la serie The Wire) dando un comunicado sobre su enfermedad por COVID-19, y por otro lado las primeras cifras de muertos por raza, que mostraban enormes desigualdades en las primeras ciudades afectadas por el virus. Desde que los primeros datos sobre la distribución geografía y social de los casos y muertes por COVID-19 empezaron a estar disponibles, quedó muy claro que se iban a reproducir los mismos patrones de desigualdades sociales en salud que llevamos décadas observando. Las primeras noticias sobre el tema en abril y mayo ya eran alarmantes. Mientras que solo un $20 \%$ de los condados del país tienen una población mayoritariamente negra, estos condados tenían más de la mitad de los casos de todo el país ${ }^{17}$. En aquel momento, en los estados que ya habían sacado datos de mortalidad, la población negra representaba el $13 \%$ del total, pero el $27 \%$ de todos los muertos por COVID1918. En la ciudad de Milwaukee, en el estado de Wisconsin, la población negra representa el $27 \%$ de la población, y un $70 \%$ de las muertes por COVID-19 ${ }^{19}$. En la Figura 5 mostramos las tasas de mortalidad por edad y raza/etnia para todo el

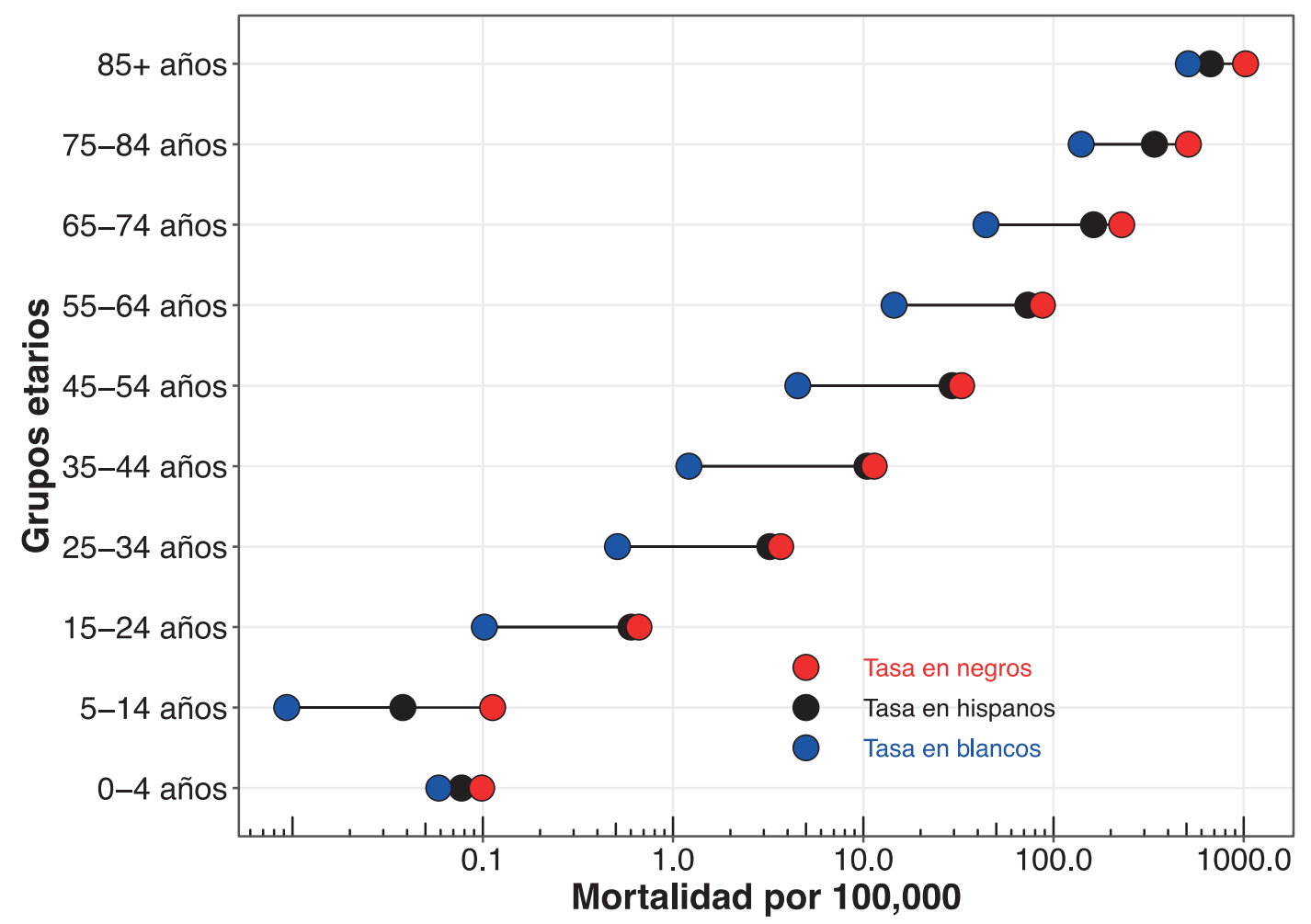

Figura 5. Mortalidad por COVID-19 por edad y raza/etina Fuente: National Center for Health Statistics ${ }^{20}$ 
país. Como podemos ver, en cada grupo etario la mortalidad de las minorías es mucho mayor que la de la población blanca.

En las grandes ciudades la situación es similar. En la Figura 6 mostramos las tasas de incidencia, hospitalizaciones y mortalidad por COVID-19, todas ellas ajustadas por edad, para varios grupos étnicos en Nueva York y Filadelfia. Como podemos ver estas tasas son de 2 a 4 veces más altas en la población negra y latina comparada con la población blanca.

Otro eje desde donde se vertebra esta desigualdad social es el eje espacial. Ante la enorme segregación racial y económica que existe en las ciudades de EEUU, esperamos que cualquier patrón social quede reflejado en un patrón espacial. Con datos disponibles a nivel de código postal en bastantes ciudades, hemos podido

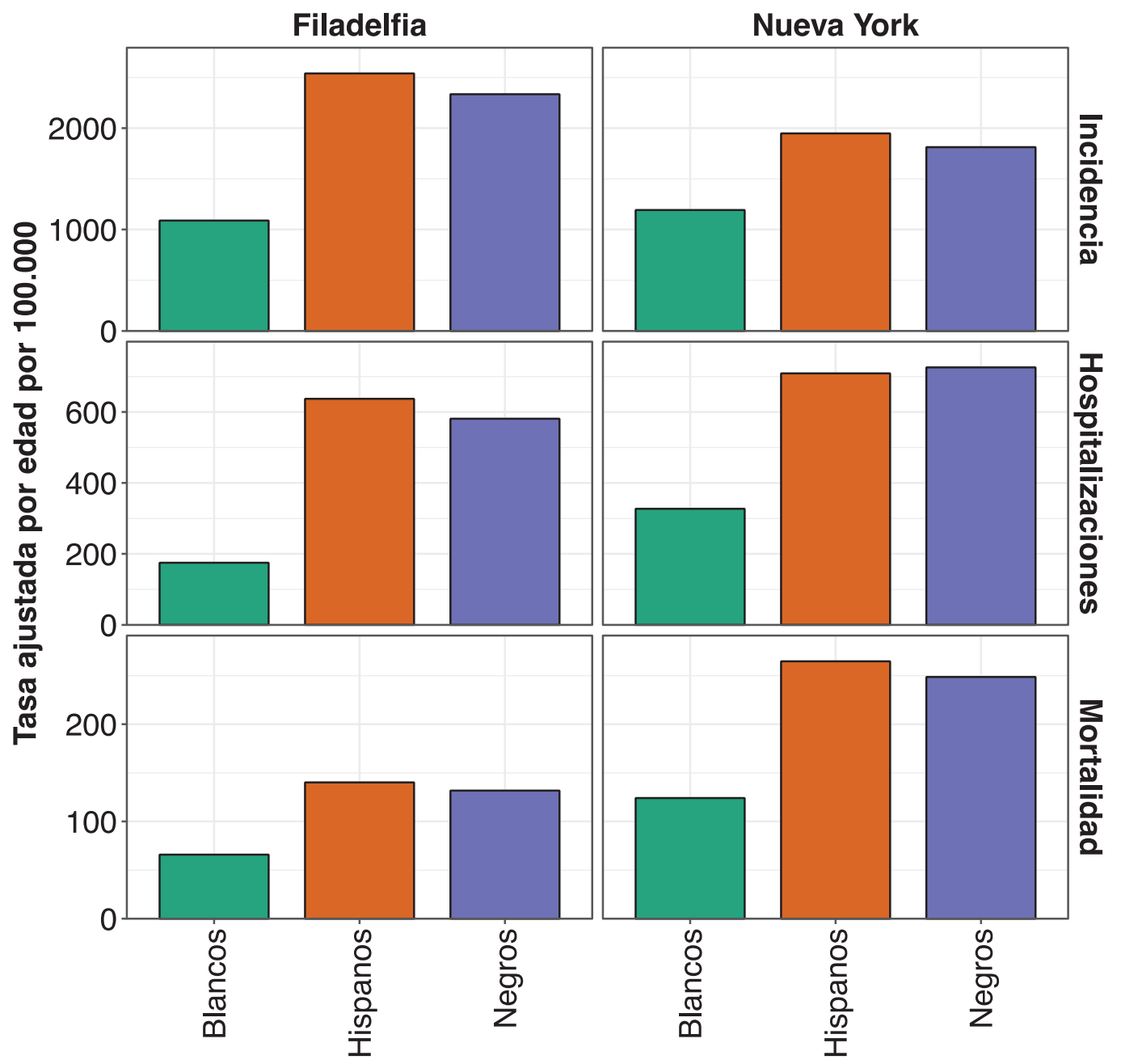

Figura 6. Desigualdades en resultados COVID-19 por raza/etnia en Filadelfia y Nueva York Fuente: Departamento de Salud Pública de Filadelfia ${ }^{21}$ y Departamento de Salud de la ciudad de Nueva York ${ }^{22}$ 
estudiar como se distribuye la carga de enfermedad y de mortalidad según otras variables de área. En la Figura 7 mostramos como se distribuye el porcentaje de pruebas positivas en cada código postal de Chicago, Filadelfia y Nueva York, según valores del índice de vulnerabilidad social, un marcador de vulnerabilidad espacial creado por el Centro de Control de Enfermedades (CDC). Como evidencia la figura, unos mayores índices de vulnerabilidad social se asocian a un mayor numero de pruebas positivas.

Este marcador, la proporción de pruebas positivas, es un marcador de enorme utilidad. Por un lado, nos da una idea de la carga de enfermedad (pruebas positivas, el numerador), pero las normaliza por la intensidad de pruebas en la zona (total de pruebas, el denominador). Esto nos permite evaluar si en una zona se están haciendo un numero suficiente de pruebas. Aquí esta otra de las desigualdades que se observó en la pandemia en EEUU, especialmente en las primeras semanas: la baja disponibilidad de pruebas, especialmente en las zonas más pobres. De hecho, una de las investigaciones que realizamos sobre este fenómeno en Filadelfia ${ }^{25}$, fue reseñada por el periódico local de Filadelfia (el Philadelphia Inquirer) y ha sido una de las pruebas postuladas por el Departamento de Salud Pública de Filadelfia para justificar la necesidad de crear una comisión de equidad racial en la respuesta al COVID-19. Este fenómeno no fue único de Filadelfia, y recientes investigaciones muestran que la falta de lugares cercanos donde realizarse las pruebas para el virus puede ser una barrera enorme para la población ${ }^{26}$.

Otros lugares donde la pandemia ha tenido un enorme impacto son los entornos donde se encuentran muchas personas, incluyendo las residencias de la tercera edad, las prisiones, y los

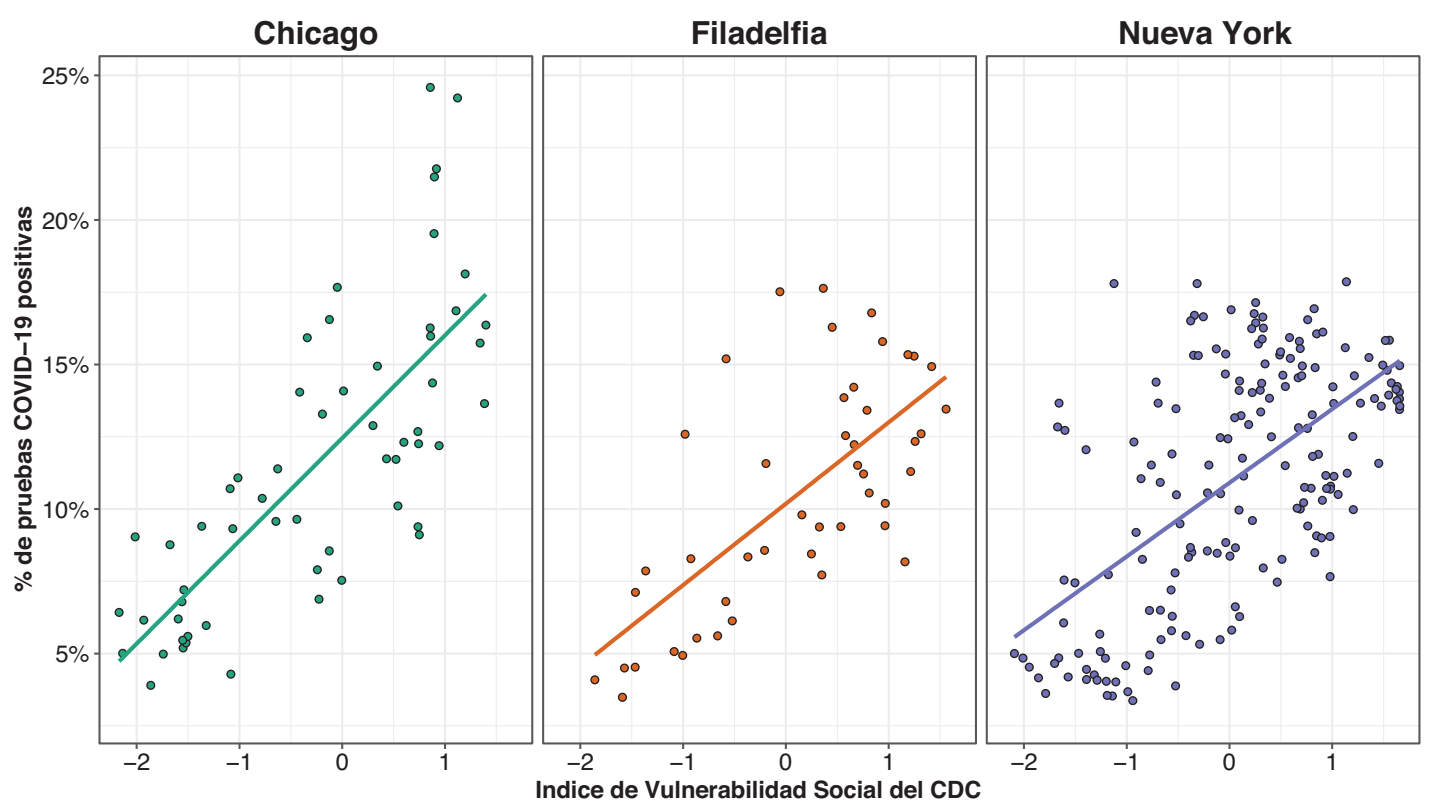

Figura 7. Vulnerabilidad social y pruebas positivas en códigos postales de 3 ciudades Fuente: Departamento de Salud Pública de Filadelfia ${ }^{21}$, Departamento de Salud de la ciudad de Nueva York ${ }^{22}$, Departamento de Salud Pública de Chicago ${ }^{23}$, y $\mathrm{CDC}^{24}$ 
centros de internamiento de inmigrantes. Al igual que en España, el impacto en las residencias de EEUU ha sido enorme. Por ejemplo, el $50 \%$ de las muertes en Filadelfia han ocurrido en centros de mayores ${ }^{27}$. En un reciente artículo publicado en Health Affairs, unos investigadores exploraron la mortalidad en residencias de la tercera edad dependiendo de si el personal de la residencia estaba afiliado a un sindicato o no ${ }^{28}$. En EEUU estas afiliaciones se hacen por centros de trabajo, creando enormes diferencias entre centros en cuanto a los derechos laborales de los trabajadores. Los autores encontraron que aquellas residencias cuyos trabajadores estaban afiliados a un sindicato tenían un $30 \%$ de mortalidad menos que las residencias donde este no era el caso $^{28}$, y que parte de esta asociación era debida a la mayor disponibilidad de equipos de protección individual para los trabajadores. Las prisiones y centros de internamiento de inmigrantes también han supuesto centros de alta transmisión, debido al hacinamiento, falta de protección y de pruebas. De hecho, los mayores brotes del país están asociados a prisiones o centros de internamiento, y en varias ocasiones los condados con mayor incidencia de la enfermedad en todo el país eran los condados con las prisiones más grandes o más afectadas por el virus. En el país que más gente encarcela del mundo, esta altísima incidencia en prisiones supone un reto enorme para el control de la pandemia, especialmente por las enormes diferencias raciales y económicas en la población encarcelada.

\section{LA SITUACIÓN POLÍTICA DURANTE LA PANDEMIA}

Por último, quiero mencionar una serie de retos a los que se ha enfrentado y sigue enfrentándose EEUU con respecto a la pandemia: (1) los problemas del sistema de salud pública; (2) los problemas del sistema sanitario; y (3) la partidización de la respuesta a la pandemia.

La respuesta del sistema de salud pública ha estado condicionada al débil rol que tiene el
Centro de Control de Enfermedades (CDC por sus siglas en ingles) en coordinar la respuesta. EEUU es un país con un sistema federal, donde los 50 estados tienen un enorme grado de autonomía en todos los aspectos que la constitución no le confiere al gobierno federal. Por ello, muchas de las decisiones sobre la respuesta recaen sobre estos estados. Idealmente, el CDC coordinaría estas decisiones, asistiría técnicamente a los estados, los ayudaría a crear protocolos, recolectaría centralmente datos, etc. En la practica, el CDC ha estado ausente o ha sido ignorado. La recolección de datos entre estados se realizo por parte de voluntarios, con esfuerzos como el panel de Johns Hopkins o el panel de COVID Tracking. El CDC no empezó a mostrar cifras oficiales hasta mayo y junio. Por ejemplo, la primera base de datos de casos, similar a la que países como México o Colombia proveen desde hace meses, no se publicó hasta finales de junio, tras una petición de transparencia del periódico New York Times. Entre otros fiascos, el CDC intentó publicar una guía de reapertura de colegios e instituciones educativas, que rápidamente fue retirada de su web al contradecir los mensajes oficiales que emitía la presidencia del país ${ }^{29}$. Los artículos en la revista MMWR (Mortality and Morbidity Weekly Reports, la revista principal de vigilancia epidemióloga del $C D C$ ) deben pasar por el filtro de una persona de confianza del presidente Trump, para evitar que salgan mensajes "políticos", incluyendo si los niños tienen más o menos probabilidad de transmitir el virus ${ }^{29}$. En septiembre mismo, durante unas horas la web del CDC reconocía la importancia de la transmisión vía aerosoles, una información que fue retirada horas más tarde de la misma pagina web ${ }^{29}$.

Con este panorama, las decisiones han quedado en manos de los estados, resultando en una enorme heterogeneidad en la respuesta. Por ejemplo, en agosto. Massachusetts, declaró una cuarentena para viajeros al estado, con multas de hasta $\$ 500$ por día de incumplimiento. Al mismo 
tiempo, Nueva Jersey tenía una cuarentena voluntaria y muchos otros estados ni siquiera tienen tal cuarentena. En otros casos, los condados y las ciudades han tenido capacidad de respuesta. Por ejemplo, la ciudad de Filadelfia ha realizado una reapertura más lenta que el estado de Pennsylvania donde esta situada, retrasando la apertura del interior de bares y restaurantes varios meses. Pero en lugares como el estado de Georgia, el propio gobierno estatal ha prohibido a las ciudades realizar este tipo de cambios, forzándolas a dejar abrir el interior de la hostelería.

Esta fragmentación del sistema de salud pública entre diferentes niveles de gobernanza tiene su correlato en el sistema sanitario, donde la fragmentación se vertebra en ejes publico privados. Un 55 \% de la población tiene aseguramiento privado, seguida de un $35 \%$ con aseguramiento publico (principalmente la gente más pobre, con discapacidades, niños, o gente mayor de 65 anos) y un $9 \%$ de gente sin seguro medico ${ }^{30}$. La provisión también varia, parte privada (sin y con animo de lucro) y pública. Esta compleja red crea enormes complicaciones para la asistencia, dado que existen infinidad de combinaciones de aseguramiento-proveedores que crean limitaciones al acceso. Por ejemplo, a pesar de que según la ley de emergencia aprobada en marzo (CARES act) todas las pruebas para el virus deben de ser gratuitas, independientemente del aseguramiento o no, existen muchos proveedores que solo las realizan a gente con seguro. También existen un gran numero de proveedores que no aceptan Medicaid, el seguro del que depende la población más pobre o con discapacidades. Por último, y dado que la grandísima parte del aseguramiento privado depende del empleo, se están creando enormes bolsas de gente sin seguro medico al haber perdido su empleo. Con 20 millones de nuevos desempleados, que reciben unas prestaciones muy limitadas y que serán más limitadas al acabarse los incentivos de la CARES act, en el contexto de los ataques permanentes a la reforma sanitaria desarrollada por Obama, no parece que la situación vaya a mejorar próximamente.

El último reto de respuesta es la partidización de las medidas. Desde un principio, con el fin de minimizar la importancia de la pandemia, el presidente atacó a los estados que pretendían tomar las medidas más restrictivas, llegando a decir que el país debía reabrirse en semana santa (ide 2020!). Estos ataques, sumados al impacto pequeño de la pandemia en aquel momento en los estados del sur, crearon un clima de desconfianza entre parte de la población con respecto a la necesidad de medidas estrictas de control. Esto llevó a manifestaciones por la reapertura, contra el uso de mascarillas, etc., muy similares a las manifestaciones que se vivieron en España. Quizás la diferencia más importante es que estas manifestaciones estaban sutilmente, o no tan sutilmente, apoyadas por el presidente del país, haciendo todavía más difícil la labor de los estados que querían mantener estas medidas. Uno de los ejemplos de las consecuencias de esta falta de medidas ocurrió en agosto, con el encuentro de moteros de Sturgis. Este encuentro congrega cada año a medio millón de personas en Dakota del Sur. Las autoridades estatales no hicieron nada por prevenir que el encuentro ocurriera, las autoridades locales no tenían competencia, y al final más de 460.000 personas, provenientes del $61 \%$ de los condados de todo EEUU, estuvieron en el encuentro, la mayoría sin mascarillas. En un estudio publicado hace unas semanas, se estimo que más de 250.000 casos observados en agosto pueden trazarse a gente que estuvo en el encuentro o a sus contactos ${ }^{31}$. La gobernadora del estado de Dakota del Sur critico el estudio como un "ataque a las libertades personales de los asistentes".

\section{CONCLUSIÓN}

El COVID-19 ha tenido un enorme impacto en Estados Unidos, ocasionando una crisis de mortalidad en ciudades como Nueva York que 


\section{COVID-19 EN ESTADOS UNIDOS: LA DESIGUALDAD SOCIAL COMO CAUSA Y CONSECUENCIA \\ USAMA BILAL}

no se veía desde la pandemia de gripe de 1918, agravando las enormes desigualdades en salud que ya existían, y poniendo de relieve la fragilidad de un sistema de salud pública y sanitario que no es sino un paralelo de la fragilidad de su casi inexistente sistema de protección social. Mi gran esperanza es que la visibilización de estas desigualdades y la evidencia de lo poco robusto de la respuesta ante una pandemia, supongan un catalizador para enormes reformas estructurales. Con las elecciones de noviembre de 2020 veremos si el país se encamina hacia alguna de estas reformas, o hacia una profundización de la brecha social y de las carencias de su sistema de protección social.

\section{REFERENCIAS}

1. Johns Hopkins University \& Medicine. COVID-19 Dashboard. 2020. 22 de septiembre de 2020. [Consultado el 22 de Septiembre de 2020].

2. OECD. Income inequality. [Internet]. 2020. [Consultado el 22 de Septiembre de 2020].

3. United Nations Department of Economic and Social Affairs. World population prospects 2019: Highlights. New York (US): United Nations Department for Economic and Social Affairs; 2019.

4. Montez JK, Beckfield J, Cooney JK, Grumbach JM, Hayward MD, Koytak SZ, et al. US State Policies, Politics, and Life Expectancy. Milbank Q 2020;98(3):668-99.

5. Knapp EA, Bilal U, Dean LT, Lazo M, Celentano DD. Economic insecurity and deaths of despair in US counties. Am J Epidemiol 2019;188(12):2131-9.

6. Bilal U, Knapp EA, Cooper RS. Swing voting in the 2016 presidential election in counties where midlife mortality has been rising in white non-Hispanic Americans. Soc Sci Med. 2018;197:33-8.

7. Hannah-Jones N, Elliott MN. The 1619 Project. New York Times 2019. [Internet]. [Consultado el 22 de Septiembre de 2020].
8. Rothstein R. The Color of Law: A Forgotten History of How Our Government Segregated America. New York: Liveright; 2017.

9. McIntosh K, Moss E, Nunn R, Shambaugh J. Examining the Black-white wealth gap. The Brookings Institution [Internet]; 2020.

10. Faust JS, Lin Z, del Rio C. Comparison of Estimated Excess Deaths in New York City During the COVID-19 and 1918 Influenza Pandemics. JAMA Network Open 2020;3:e2017527-e.

11. Trias-Llimos S, Riffe T, Bilal U. Monitoring life expectancy levels during the COVID-19 pandemic: Example of the unequal impact in Spanish regions. medRxiv [Internet]. 2020:2020.06.03.20120972.

12. MoMo. Sistema de Monitorización de la Mortalidad diaria. [Internet]. 2020. [Consultado el 22 de Septiembre de 2020].

13. Centers for Disease Control and Prevention. Excess Deaths Associated with COVID-19. [Internet]. 2020. [Consultado el 22 de Septiembre de 2020].

14. Cevik M, Marcus JL, Buckee C, Smith TC. SARSCoV-2 transmission dynamics should inform policy. CID 2020 ciaa1442. (En prensa).

15. GitHub. Coronavirus (Covid-19) Data in the United States. [Internet]. 2020. [Consultado el 22 de Septiembre de 2020].

16. Wamsley L. Fired Florida Data Scientist Launches A Coronavirus Dashboard Of Her Own. NPR [Internet]. 14 de junio de 2020.

17. Millett GA, Jones AT, Benkeser D, Baral S, Mercer L, Beyrer C, et al. Assessing Differential Impacts of COVID-19 on Black Communities. Ann Epidem 2020; 47: 37-44.

18. APM Research Lab. The color of coronavirus: COVID-19 deaths by race and ethnicity in the US. [Internet]. 2020. [Consultado el 22 de Septiembre de 2020].

19. Rast J. Milwaukee's Coronavirus Racial Divide: A Report on the Early Stages of COVID-19 Spread in Milwaukee County: University of Wisconsin-Milwaukee; 2020. 


\section{COVID-19 EN ESTADOS UNIDOS: LA DESIGUALDAD SOCIAL COMO CAUSA Y CONSECUENCIA USAMA BILAL}

20. Centers for Disease Control and Prevention. Deaths involving COVID-19 2020. [Internet]. 2020. [Consultado el 22 de Septiembre de 2020].

21. City of Philadelphia. COVID-19 Testing and data. 2020. [Internet]. 2020. [Consultado el 22 de Septiembre de 2020].

22. GitHub. NYC Coronavirus (COVID-19) data. [Internet]. 2020. [Consultado el 22 de Septiembre de 2020].

23. Chicago Data Portal. COVID-19 Cases, Tests, and Deaths by ZIP Code. [Internet]. 2020. (Consultado el 22 de Septiembre de 2020).

24. Flanagan BE, Hallisey EJ, Adams E, Lavery A. Measuring Community Vulnerability to Natural and Anthropogenic Hazards: The Centers for Disease Control and Prevention's Social Vulnerability Index. J Environ Health 2018;80(10):34-6.

25. Bilal U, Barber S, Diez-Roux AV. Spatial Inequities in COVID-19 outcomes in Three US Cities. medRxiv. 2020:2020.05.01.20087833.

26. Rader B, Astley CM, Sy KTL, Sewalk K, Hswen Y, Brownstein JS, et al. Geographic access to United States SARS-CoV-2 testing sites highlights healthcare disparities and may bias transmission estimates. J Travel Med 2020;taaa076. (En prensa).

27. City of Philadelphia. Ciudad ofrece actualización sobre el COVID-19 para el 18 de septiembre de 2020. [Internet]. 2020. [Consultado el 22 de Septiembre de 2020].

28. Dean A, Venkataramani A, Kimmel S. Mortality Rates From COVID-19 Are Lower In Unionized Nursing Homes: Study examines mortality rates in New York nursing homes. Health Aff 2020; 39:11.

29. Avril T. As CDC reverses itself on COVID-19, Americans are losing trust, warn public-health veterans. The Philadelphia Inquirer. [Internet]. 22 de septiembre de 2020. [Consultado el 22 de Septiembre de 2020].

30. KFF. Health Insurance Coverage of the Total Population. [Internet]. 2018. [Consultado el 22 de Septiembre de 2020].

31. Dave DM, Friedson Al, McNichols D, Sabia JJ. The Contagion Externality of a Superspreading Event: The Sturgis Motorcycle Rally and COVID-19: National Bureau of Economic Research;. Report No.: 0898-2937. IZA - Institute of Labor Economics [Intenet] septiembre de 2020

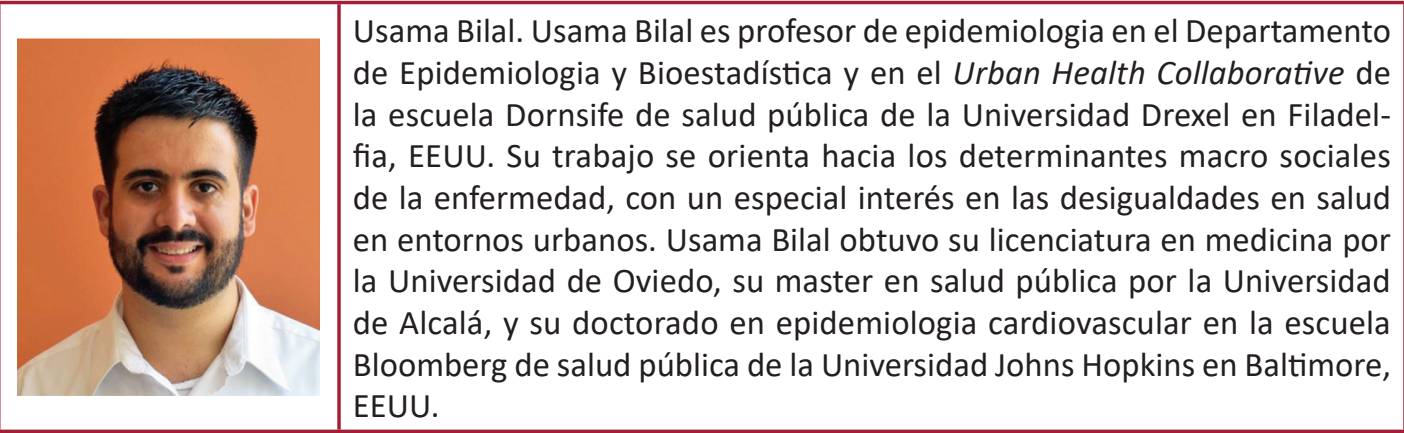

Rev. Med. Cine. 2020; 16 (e), 105-117 Ediciones Universidad de Salamanca / @®@@ J. Med. Mov., 2020; 16 (e), 105-117 [117] 